\title{
Expression of the Aspergillus nidulans argB Gene in Escherichia coli
}

\author{
By ALEKSANDRA DMOCHOWSKA, BRYGIDA BERSE, \\ IWONA KOWALSKA, MAREK SKRZYPEK \\ AND PIOTR WĘGLEŃSKI* \\ Department of Genetics, Warsaw University, Al. Ujazdowskie 4, 00-478 Warsaw, Poland
}

(Received 28 October 1985)

\begin{abstract}
The Aspergillus nidulans $\arg B$ gene coding for ornithine carbamoyltransferase (OTCase) is not expressed in Escherichia coli. However, E. coli OTCase-deficient strains transformed with plasmids carrying the $\arg B$ gene from $A$. nidulans reverted to prototrophy at a high frequency. In these derivatives the $\arg B$ gene became functional due to DNA rearrangements upstream of the coding sequence. Two types of rearrangement were characterized. One was identified as an insertion of IS2. The second was a deletion that resulted in transcription of the $\arg B$ gene from the $\mathrm{Tc}^{\mathrm{R}}$ gene promoter and translation from a newly created ribosome-binding site formed at the junction between the $A$. nidulans and vector DNA sequences.
\end{abstract}

\section{INTRODUCTION}

Attempts to clone eukaryotic genes by complementation of bacterial mutations have been successful only in the case of lower eukaryotes, in which most genes lack introns. Only a limited number of genes have been cloned in this way, indicating that the expression of such genes in bacterial cells is the exception rather than the rule. This is certainly true for genes of the filamentous fungus $A$ spergillus nidulans. We were interested in cloning the $A$. nidulans $\arg B$ gene coding for ornithine carbamoyltransferase (OTCase), the key enzyme in the arginine biosynthetic pathway. Despite many efforts we were unable to clone this gene (as well as several other $A$. nidulans genes) in Escherichia coli directly. We finally succeeded in cloning it in yeast and identified it by complementation of the appropriate yeast mutation (Berse et al., 1983). Introduction of the $\arg B$ gene into an OTCase-deficient $E$. coli strain did not result in recovery of prototrophy by the transformed cells. However, strains bearing plasmids containing the $\arg B$ gene reverted to prototrophy at a high frequency. The revertants produced OTCase with the immunological properties of the $A$. nidulans enzyme, and they yielded plasmids capable of rendering OTCase-deficient $E$. coli mutants prototrophic. These plasmids differed in size from the original one as well as among themselves, indicating that structural rearrangements had made expression of the $\arg B$ gene possible.

We believed that a comparison of the structure of the original plasmid with its derivatives, together with an analysis of their transcription and translation products, would yield information on the barriers preventing expression of the $\arg B$ gene in the bacterial host and on the mechanisms by which these barriers could be overcome. In this paper we present results from an analysis of three different plasmids containing the $\arg B$ gene expressed in $E$. coli and describe the character of the changes which made this gene functional.

\section{METHODS}

Micro-organisms and growth conditions. The E. coli argF argI mutant used in this study was C600 OTC $^{-}$Thi-HsdR-HsdM- $^{-} \Delta$ (pro-lac) argIl (Crabeel et al., 1979). Plasmids were propagated in E. coli strain JA221 (Beggs, 1978). The minicell-producing strain $\chi 1849$ [tonA53 T1 ${ }^{\circ}$ dapD8 $\operatorname{minAl}$ purEl supE42 $\Delta\left(\right.$ gal-uvrB) $\lambda^{-} \min B 2$

Abbretiation: OTCase, ornithine carbamoyltransferase. 
his-53 nal-25 metC65 T $3^{r} \Delta($ bioH-asd) ilv-227 cycB2 cycAl hsdR2] and bacteriophage $\lambda$ cI857 were obtained from the collection of the Department of Virology, Warsaw University. Bacteria were cultivated in L-broth or in M9 medium (Miller, 1972). Saccharomyces cerevisiae strain SPU1 carrying the arg3 mutation, and yeast growth conditions, were described by Berse et al. (1983).

Plasmids. The vectors used were pBR327 (Soberon et al., 1980) and pBB29 (Berse et al., 1983). Plasmid pLK5 containing the phage $\lambda P_{R}$ promoter (Zabeau \& Stanley, 1982) was obtained from EMBL, Heidelberg, FRG. Plasmids pSal43 and pSal43* are derivatives of plasmid pBBl 16 described by Berse et al. (1983). They contain a $2.6 \mathrm{~kb}$ fragment of $A$. nidulans DNA with the complete $\arg B$ gene which is functional in yeast. The $A$. nidulans DNA is inserted in opposite orientations into the $S a l$ site located within the Tc ${ }^{R}$ gene of $\mathrm{pBB} 29$ (Fig. 1). Plasmids pSal23 and pSal23* do not contain yeast sequences within the vector DNA. They were obtained by removal of two small EcoRI fragments from pSal43 and pSal43*, respectively. Derivatives of plasmids pSal43-5, pSal43*-3 and pSal43*-4 (described in Results) were obtained in an analogous way and designated pSal23-5, pSal23*-3 and pSal23*-4, respectively.

Transformation. E. coli was transformed as described by Dagert \& Ehrlich (1979). Yeast transformation was performed according to the method of Hinnen et al. (1978).

DNA isolation. Plasmid DNA was isolated by the technique of Clewell \& Helinski (1969) and purified by caesium chloride/ethidium bromide centrifugation. Total $E$. coli DNA was extracted by the method described by Cosloy \& Oishi (1973). DNA fragments were recovered from agarose or polyacrylamide gels according to Maxam \& Gilbert (1977).

Blot hybridization. DNA-DNA hybridization was done by the method of Southern (1975). DNA-RNA hybridization was performed according to Thomas (1980). DNA used as a probe was labelled with $\left[\alpha{ }^{-32} \mathrm{P}\right] \mathrm{dATP}$ or $\left[\alpha^{-32} \mathrm{P}\right] \mathrm{dCTP}$ by nick-translation as described by Mackey et al. (1977).

In vitro transcription. The reaction was carried out following Lee \& Yanofsky (1977) with slight modifications. The reaction mixture $(50 \mu \mathrm{l})$ contained $5 \mu \mathrm{g}$ plasmid DNA, 2.5 units of RNA polymerase and each nucleotide triphosphate at a concentration of $0.2 \mathrm{~mm}$. Transcripts purified by DNAase I treatment and phenol extraction were separated in an agarose gel according to Lehrach et al. (1977).

Analysis of protein synthesis in minicells. E. coli minicells were purified following the procedure of Reeve (1979). Proteins synthesized in minicells were labelled using a ${ }^{14} \mathrm{C}$-labelled amino acids mixture as described by Roozen $e t$ al. (1971). Proteins were separated in $12.5 \%(\mathrm{w} / \mathrm{v})$ polyacrylamide-SDS gels according to Laemmli (1970). Fluorography was done by the method of Bonner \& Laskey (1974).

OTCase assay. OTCase activity was assayed according to Cybis et al. (1972).

DNA sequencing. The fragment of pSal43-5 was sequenced by the method of Maxam \& Gilbert (1980). In the case of pSal43*-3, M13 cloning and sequencing by the Sanger method were done according to the Amersham manual.

\section{RESULTS}

Selection of plasmids with the A. nidulans argB gene expressed in $E$. coli

The $E$. coli double mutant $\arg F \operatorname{argl}$, which lacks OTCase activity, was transformed with plasmids pSal43 and pSal43*, which carry the $A$. nidulans $\arg B$ gene in opposite orientations with respect to the vector (see Fig. 1). The transformants required arginine for growth but reverted to prototrophy at a frequency of $10^{-3}$ in the case of pSal43 and of $10^{-5}$ in the case of pSal43*. Plasmid DNA isolated from seven $\mathrm{Arg}^{+}$colonies was analysed by agarose gel electrophoresis. Six out of seven plasmids differed significantly in size from the parental plasmid and three of them were investigated further. The first plasmid (named pSal43-5) originated from pSal43 and was smaller than it. Two others (pSal43*-3 and pSal43*-4) were derived from pSal43* and were bigger than it. All three derivative plasmids were able to suppress the $\arg F$ and $\arg I$ mutations in E. coli, and they retained the ability to complement the $\arg 3$ mutation in yeast. Yeast DNA sequences were removed from the vector part of pSal43, pSal43* and their derivatives, to yield a series of corresponding plasmids (see Methods). As expected, plasmids pSal23-5, pSal23*-3 and pSal23*-4, like their counterparts pSal43-5, pSal43*-3 and pSal43*-4, suppressed the $\arg I$ and $\arg F$ mutations in $E$. coli. The activity of the OTCase in $E$. coli strains bearing these plasmids was substantially higher than that in strains carrying plasmids pSal23 and pSal23*, which carry the $\arg B$ gene in its original cloned form (Table 1). It is worth noting that the OTCase activity present in the $E$. coli strains bearing pSal23 and pSal23* is not sufficient to allow the growth of these strains on the arginine-free medium. The enzyme was very stable in $E$. coli, as the activity showed no decrease after $48 \mathrm{~h}$ incubation of bacteria in the presence of chloramphenicol. 

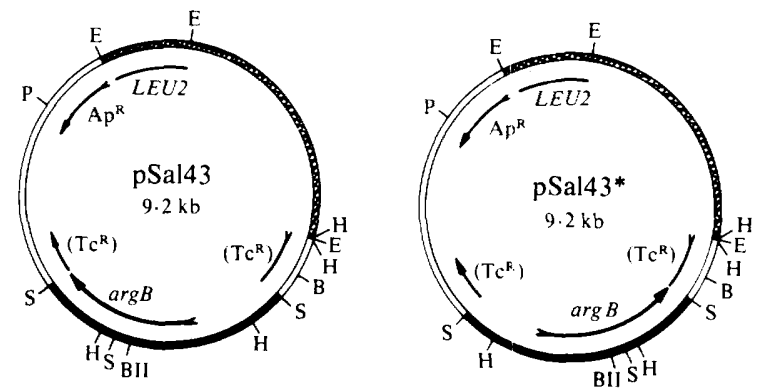

Fig. 1. Restriction maps of pSal43 and pSal43*.pBR327 sequences are represented by the double open lines, $A$. nidulans DNA by the solid bars and yeast DNA by the hatched areas. The position of the $\arg B$ gene within the $A$. nidulans DNA is indicated. Abbreviations: B, BamHI; BII, Bg/II; E, EcoRI; H, HindIII; P, PstI; S, Sall.

Table 1. OTCase activity in E. coli strains carrying plasmids with the A. nidulans argB gene

\begin{tabular}{|c|c|c|c|}
\hline \multirow[b]{2}{*}{ E. coli strain } & \multirow[b]{2}{*}{ Plasmid } & \multicolumn{2}{|c|}{ OTCase activity* } \\
\hline & & M9 medium & $\begin{array}{l}\text { M9 medium with } \\
\text { arginine }\left(10 \mu \mathrm{g} \mathrm{ml}^{-1}\right)\end{array}$ \\
\hline $\begin{array}{l}\text { C600 OTC }- \\
(\arg F \text { argI mutant })\end{array}$ & $\begin{array}{l}\text { pSal23 } \\
\text { pSal23* } \\
\text { pSal23-5 } \\
\text { pSal23*-3 } \\
\text { pSal23*-4 } \\
\text { pSal23-50 } \\
\text { pBR327 }\end{array}$ & $\begin{array}{c}- \\
2.82 \pm 0.41 \\
3.23 \pm 0.135 \\
1.23 \pm 0.15 \\
\ldots\end{array}$ & $\begin{array}{c}0.14 \pm 0.015 \\
0.09 \pm 0.01 \\
1.93 \pm 0.28 \\
2.37 \pm 0.41 \\
1.51 \pm 0.13 \\
0.00 \\
0.00\end{array}$ \\
\hline $\begin{array}{l}\text { JA221 } \\
\text { (wild-type) }\end{array}$ & - & $13.80 \pm 0.60$ & $0.22 \pm 0.04$ \\
\hline
\end{tabular}

* Expressed as mM-citrulline $\mathrm{h}^{-1}(\mathrm{mg} \text { protein })^{-1}$. The values are means of at least three independent assays, \pm SE.

$D N A$ insertion leading to the expression of the $A$. nidulans $\arg B$ gene in $E$. coli

The restriction patterns of $\mathrm{pSal} 43^{*}$ and its derivative $\mathrm{pSal} 43^{*}-3$ were compared. The $1.8 \mathrm{~kb}$ Sall fragment of pSal43* (see Fig. 1) was replaced by a $3 \cdot 1 \mathrm{~kb}$ fragment in pSal43*-3, indicating that a DNA sequence of about $1 \cdot 3 \mathrm{~kb}$ had been inserted into the $A$. nidulans DNA. In order to elucidate the origin of this insertion, the DNA of pSal43*-3 was hybridized to restriction fragments of total E. coli DNA. pSal43*-3 revealed strong homology to five BgllI fragments (Fig. 2, lane B). This result suggested that the extra DNA present in pSal43*-3 was derived from the $E$. coli genome and could be an IS element. IS 2 was the favoured candidate because its size is approximately that of the insert (1327 nucleotides; Ghosal et al., 1979), it occurs in five copies in the genomes of most E. coli strains (Calos \& Miller, 1980), and it is known to promote expression of adjacent genes (Kleckner, 1981). To test this supposition we used the M13 system to reclone the $0.6 \mathrm{~kb}$ HindIII fragment of pSal43*-3 containing the right-end portion of the inserted $E$. coli DNA (see Fig. 3). The sequence of the 175 nucleotides to the right of the HindlII site and within the insert was determined by the Sanger method and was found to be completely homologous with that of IS2 as reported by Ghosal et al. (1979). The IS2 element in pSal43*-3 is inserted in orientation II (Ghosal et al., 1979), about 500 nucleotides from the $5^{\prime}$ end of the $\arg B$ gene.

Another pSal43* derivative, pSal43*-4, was also hybridized to total $E$. coli DNA; it gave the same pattern of hybridization as pSal43*-3 (Fig. 2). Moreover, pSal43*-4 showed the same AluI and $M s p I$ restriction sites as pSal43*-3 (Fig. 3). This result indicated that IS2 is also present in this plasmid. However, the results of restriction mapping (not shown) revealed that, besides the IS2 insertion, other rearrangements had occurred in this plasmid DNA.pSal43*-4 was a doublet 


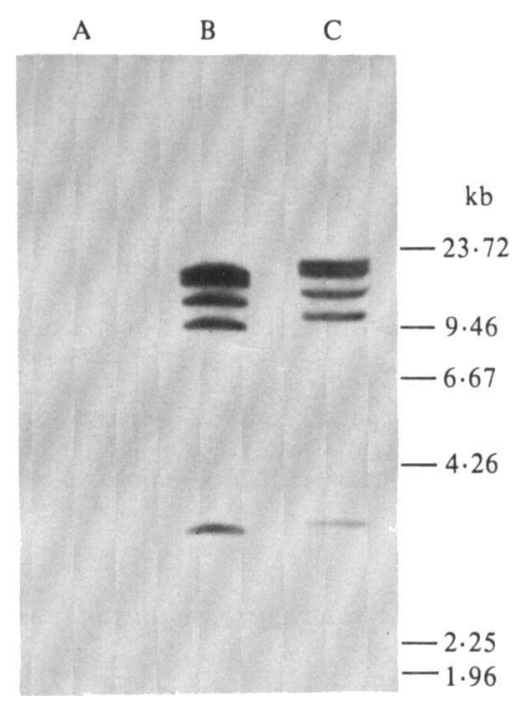

Fig. 2. Autoradiogram of Southern blots of restriction fragments of $E$. coli genomic DNA probed with DNA of pSal43* and its derivatives. The $E$. coli DNA was digested with $B g l \mathrm{II}$, transferred onto nitrocellulose and hybridized to ${ }^{32}$ P-labelled DNA of pSal43* (lane A), pSal43*-3 (lane B) and pSal43*4 (lane C). Bacteriophage $\lambda$ DNA cleaved with HindIII was used as a DNA size marker.

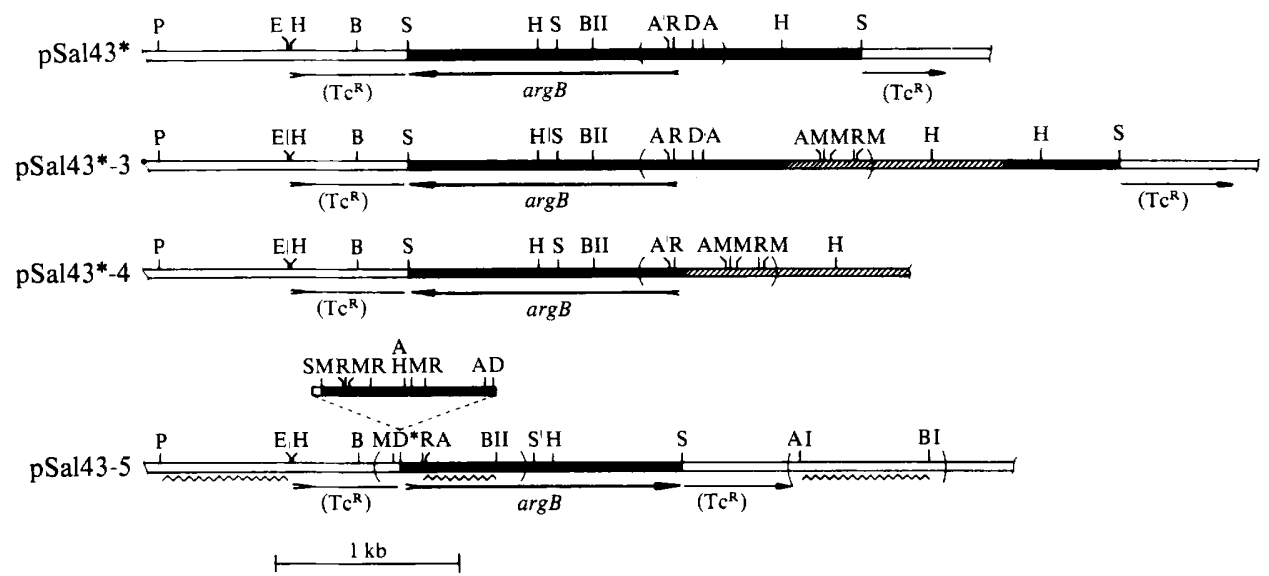

Fig. 3. Restriction maps of the A. nidulans DNA and flanking sequences within plasmids pSal43*, pSal43*-3, pSal43*-4 and pSal43-5. The portion of DNA present in pSal43 and deleted from pSal43-5 is shown above the map of the latter plasmid. The double open lines represent pBR 327 sequences, the solid bars $A$. nidulans DNA, and the hatched areas IS 2 sequences. The zig-zag lines indicate the DNA fragments used as probes in hybridization with in vitro transcription products. Abbreviations: A, AluI ; AI, AvaI ; B, BamHI; BI, BgII; BII, BglII; D, DdeI ; E, EcoRI; H, HindIII; M, MspI ; P, PstI ; R, RsaI; S, SalI. Restriction sites for $A l u \mathrm{I}, D d e \mathrm{I}, M s p \mathrm{I}$ and $R s a \mathrm{I}$ are shown only in the regions of the maps within parentheses. $\mathrm{D}^{*}$ indicates the $D$ deI site created as a result of the deletion in pSal43-5.

of pSal43* with IS2 inserted within one of the $A$. nidulans DNA fragments, and it also had a deletion of $2.5 \mathrm{~kb}$ adjacent to IS2. This plasmid contained two copies of the $\arg B$ gene, one indistinguishable from that present in pSal43* and the other with IS 2 located about 20 nucleotides from the $5^{\prime}$ end of this gene (Fig. 3). To check which copy was responsible for the expression of the $\arg B$ gene in $E$. coli, the $1.65 \mathrm{~kb}$ HindIII fragment containing part of the $\arg B$ gene and the large portion of IS2 was ligated with the $9.6 \mathrm{~kb}$ HindIII fragment of pSal43 containing the rest of the $\arg B$ gene and the vector sequences (see Figs 1 and 3). The new plasmid 


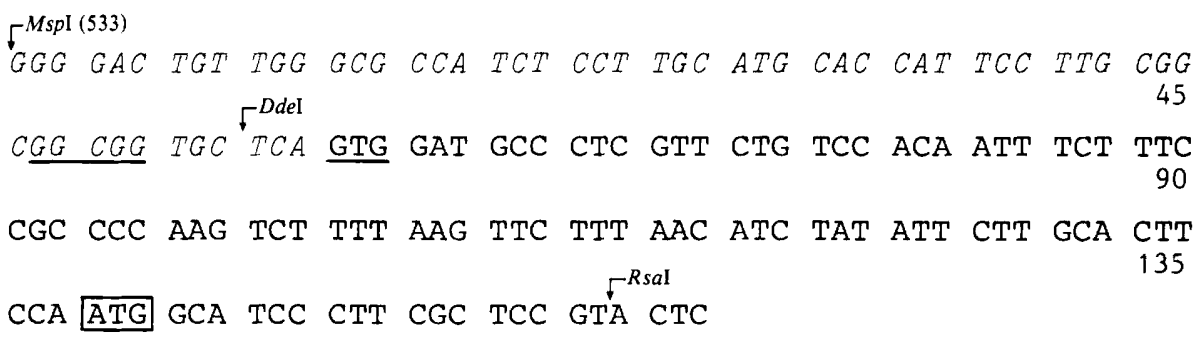

Fig. 4. Nucleotide sequence of the fragment of pSal43-5 comprising the new junction between the A. nidulans DNA and pBR327 DNA. The sequence of pBR327 (Sutcliffe, 1979) is shown in italics. The sequence begins from the $M s p I$ site at the position 533 in pBR327. The AUG postulated as the initiation site for OTCase synthesis in $A$. nidulans (B. Berse, unpublished) is boxed. The putative prokaryotic ribosome-binding site is underlined.

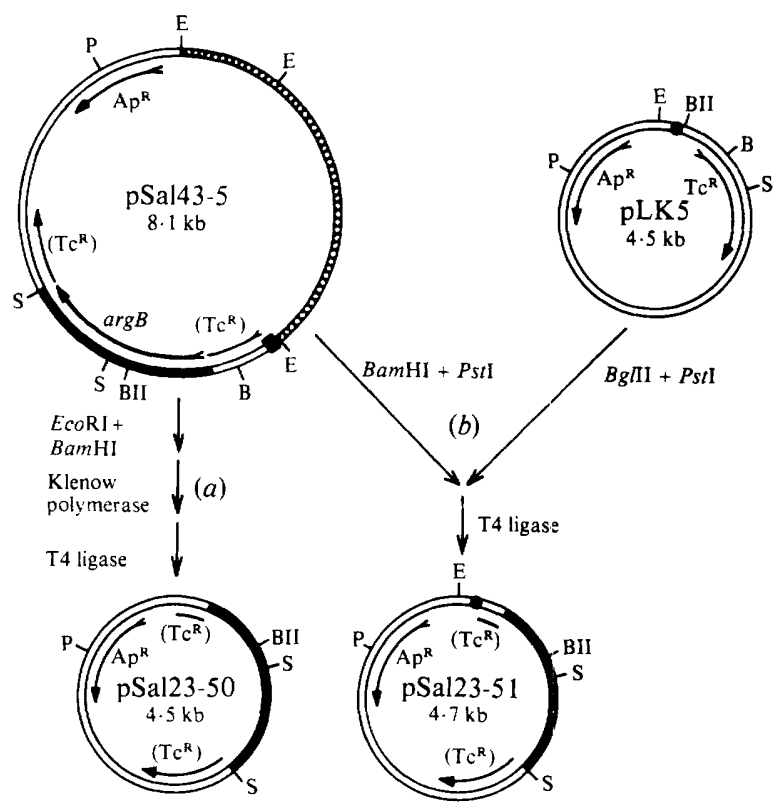

Fig. 5. Construction of plasmids pSal23-50 (a) and pSal23-5l (b). $\square$ indicates the promoter of the $\mathrm{Tc}^{\mathrm{R}}$ gene; indicates the $P_{R}$ promoter of phage $\lambda$. In plasmid pLK5 the double open line represents the pBR322 sequence. Other designations and abbreviations are as for Fig. 1.

was able to complement the $\arg F$ and $\arg I$ mutations in $E$. coli. This result proved that the expression of the $\arg B$ gene in $E$. coli bearing plasmid pSal43*-4 was connected with the presence of IS 2 in front of the coding sequence.

\section{Deletion leading to the expression of the $\arg B$ gene in $E$. coli}

Plasmid pSal43-5 was about $1 \cdot 1 \mathrm{~kb}$ smaller than the original plasmid pSal43. Comparison of the restriction patterns of these plasmids revealed that the deletion comprised sequences of the original $\mathrm{Tc}^{\mathrm{R}}$ gene and neighbouring $A$. nidulans DNA sequences of about $1 \mathrm{~kb}$ (Fig. 3). Analysis of the nucleotide sequence of the DNA fragment which included the new junction between the $A$. nidulans DNA and the vector DNA revealed that 58 nucleotides of the deleted fragment came from pBR327 and the rest (about 1100 nucleotides) from the $A$. nidulans DNA (Fig. 4).

As a result of the deletion, the $\arg B$ gene was closer to the promoter of the $\mathrm{Tc}^{\mathrm{R}}$ gene. To test whether the transcription of the $\arg B$ gene in pSal43-5 is under the control of the $\mathrm{Tc}^{\mathrm{R}}$ gene promoter, a DNA fragment containing the $5^{\prime}$ end of the $\mathrm{Tc}^{\mathrm{R}}$ gene was removed from this plasmid (Fig. 5a). The resulting plasmid (pSal23-50) was unable to suppress the $\arg F$ and $\arg I$ mutations in E. coli and no OTCase activity was detected in cells carrying this plasmid (Table 1). 


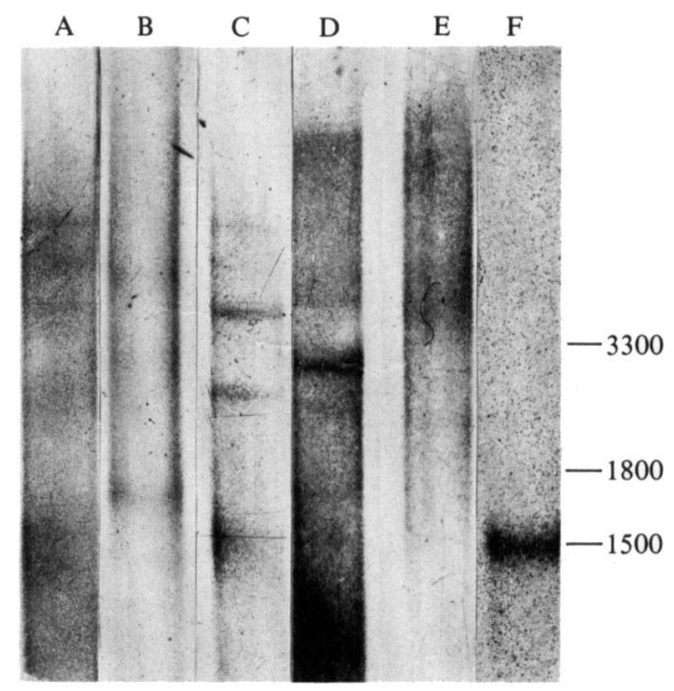

Fig. 6. In vitro transcription products of plasmids pSal23 (lanes A, B and C), pSal23-5 (lane D) and pSal23-50 (lane E) probed with the $\arg B$ gene and with DNA fragments adjacent to the $\mathrm{Tc}^{\mathrm{R}}$ gene. Transcripts were separated on a formaldehyde-agarose gel, transferred to nitrocellulose and hybridized to the following ${ }^{32} \mathrm{P}$-labelled probes: the $0.4 \mathrm{~kb}$ AluI-BgllI fragment of the $\arg B$ coding sequence (lanes $\mathrm{C}, \mathrm{D}, \mathrm{E})$, the $0.65 \mathrm{~kb}$ EcoR I-Pst I fragment adjacent to the $5^{\prime}$ end of the $\mathrm{Tc}^{\mathrm{R}}$ gene (lane A), and the $0.9 \mathrm{~kb}$ $A v a I-B g h$ fragment adjacent to the $3^{\prime}$ end of the $\mathrm{Tc}^{\mathrm{R}}$ gene (lane $\mathrm{B}$ ). The positions of these DNA fragments are shown on Fig. 3. For calibration, $A$. nidulans RNA was run on the same gel (lane F); the band corresponding to the OTCase mRNA (1500 nucleotides) is visible on the autoradiogram (the hybridization probe was the same as in lanes C, D and E). The positions of the two rRNA classes ( 3300 and 1800 nucleotides) seen on the gel are marked.

Further evidence that the $A$. nidulans $\arg B$ gene in plasmid pSal43-5 is transcribed from a prokaryotic promoter was obtained by replacing the $T \mathrm{C}^{\mathrm{R}}$ gene promoter with the $\mathrm{P}_{\mathrm{R}}$ promoter of $\lambda$ phage (Fig. 5b). The resulting plasmid (pSal23-51) was introduced into E. coli C600 OTC lysogenized with $\lambda c \mathrm{I} 857$ (a mutant with a temperature-sensitive repressor). The transformants did not grow on a medium without arginine at $30^{\circ} \mathrm{C}$, while they grew well at $37^{\circ} \mathrm{C}$. OTCase activity was 30 times higher in the cells cultivated at $37^{\circ} \mathrm{C}$ than in those grown at $30^{\circ} \mathrm{C}$. This result showed that in plasmid pSal23-51 the $A$. nidulans $\arg B$ gene is controlled by the $\lambda$ promoter.

To determine whether the transcription of the $\arg B$ gene from the bacterial promoter occurred as a result of the deletion or was already present in the original plasmid, in vitro transcripts of plasmids pSal23, pSal23-5 and pSal23-50 were analysed. The transcripts were separated by agarose gel electrophoresis under denaturing conditions, transferred onto nitrocellulose and hybridized with a ${ }^{32} \mathrm{P}$-labelled $0.4 \mathrm{~kb}$ fragment of the $\arg B$ gene (the $A l u \mathrm{I}-B g / \mathrm{II}$ fragment, see Fig. 3). Only one predominant transcript, about 3000 nucleotides in size, was synthesized from pSal23-5 (Fig. 6). This is the size expected for the transcript that begins and finishes at the appropriate signals of the $\mathrm{Tc}^{\mathrm{R}}$ gene. This transcript was not observed when pSal23-50 (which does not contain the $\mathrm{Tc}^{\mathrm{R}}$ gene promoter) was used as the DNA template. In the case of pSal23. several transcripts were obtained. One of them, about 4000 nucleotides in size, corresponds to a possible mRNA extending from the promoter to the terminator of the $\mathrm{Tc}^{\mathrm{R}}$ gene. This transcript showed no homology to pBR327 DNA fragments adjacent to the $5^{\prime}$ and $3^{\prime}$ ends of the $\mathrm{Tc}^{\mathrm{R}}$ gene (Fig. 6, lanes $\mathrm{A}$ and $\mathrm{B}$ ). It seems, therefore, that the $\mathrm{Tc}^{\mathrm{R}}$ gene promoter of pSal23 is used for transcription of the $\arg B$ gene, but the plasmid is still unable to complement the OTCase deficiency in $E$. coli, unlike its functional derivative pSal23-5. Thus the high expression of the $\arg B$ gene of plasmid pSal23-5 (and pSal43-5) is not only due to efficient transcription.

A deletion could increase the level of translation of the $\arg B$ gene by making available a ribosome-binding site present in the vector or by creating a new one. To investigate this, we 


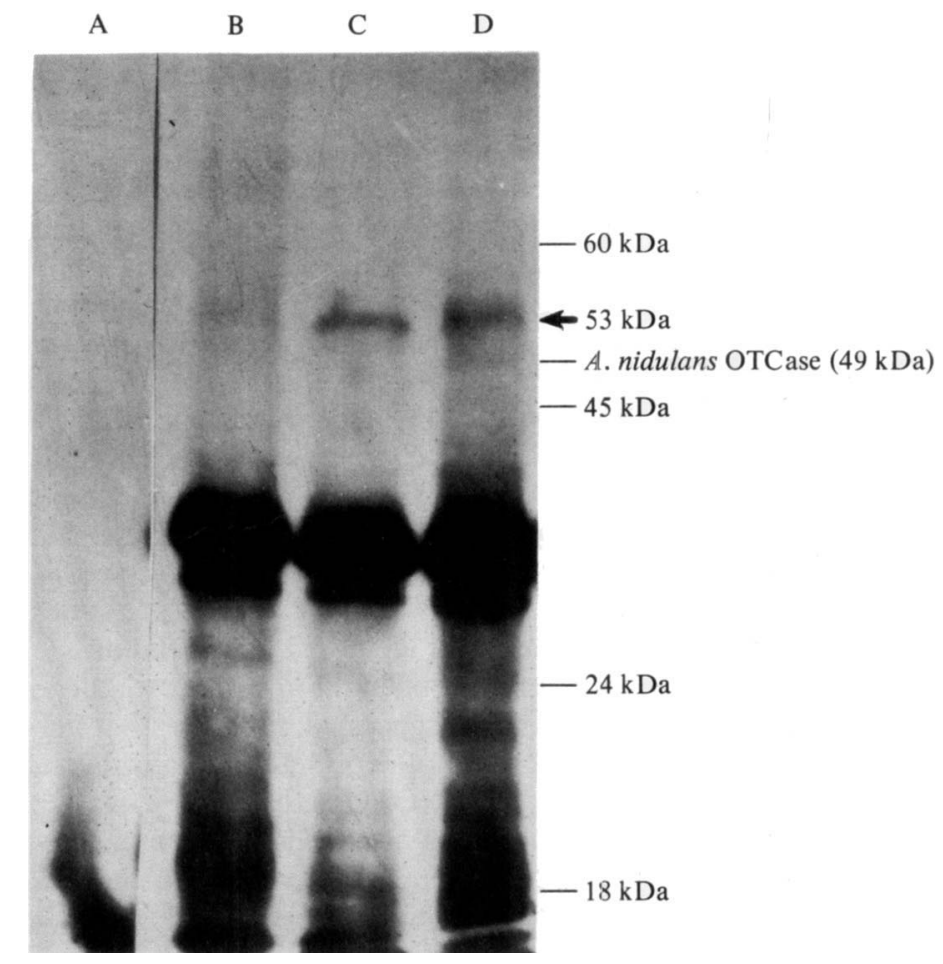

Fig. 7. Proteins synthesized in E. coli minicells carrying plasmids pSal23-50 (lane B), pSal23-51 (lane C) and pSal23-5 (lane D). ${ }^{14} \mathrm{C}$-labelled proteins were isolated from minicells, separated in an SDSpolyacrylamide gel and visualized by fluorography. Proteins isolated from minicells containing no plasmid were run in the gel as a control (lane A). The position in the gel of standards and of the OTCase isolated from $A$. nidulans mycelium is indicated. The arrow indicates the band corresponding to the $\arg B$ gene product in $E$. coli.

compared the OTCase synthesized in E. coli minicells with that present in $A$. nidulans mycelium. Strain $\lambda 1849$ was transformed separately with plasmids pSal23-4, pSal23-50 and pSal23-51 and minicells were isolated. Proteins synthesized in the minicells were labelled with ${ }^{14} \mathrm{C}$, separated on a polyacrylamide gel and visualized by fluorography. OTCase isolated from $A$. nidulans mycelium (the procedure of isolation will be published elsewhere) was run on the same gel (Fig. 7). In extracts from minicells carrying pSal23-5 or pSal23-51 one additional $53 \mathrm{kDa}$ protein band was present, which was not observed in the case of pSal23-50. We concluded that this band corresponds to the OTCase, because the only difference between pSal23-50 and the two other plasmids is the absence of a bacterial promoter for transcription of the $\arg B$ gene in the former plasmid. The OTCase detected in the minicells was about $4 \mathrm{kDa}$ larger than that isolated from $A$. nidulans mycelium, suggesting that in $E$. coli the translation of the $\arg B$ gene starts upstream of the initiation site used in $A$. nidulans. Analysis of the nucleotide sequence of this region in pSal43-5 revealed the presence of a sequence that resembles the prokaryotic ribosome-binding site (Fig. 4). This sequence comprises a GTG codon preceded (with a gap of six nucleotides) by the sequence GGCGG, which closely resembles the Shine-Dalgarno sequence GGAGG (Kozak, 1983). The proposed ribosome-binding site is located at a junction between $A$. nidulans and pBR327 DNA: the GTG initiation codon comes from the $A$. nidulans DNA and the ShineDalgarno sequence from the vector. The use of this site, created by the deletion, to initiate the translation of the $\arg B$ gene in $E$. coli could explain the efficient expression of this gene in cells carrying plasmid pSal43-5. 


\section{DISCUSSION}

In this paper we have described the characterization of two types of DNA rearrangements that resulted in the activation of the $\arg B$ gene from $A$. nidulans in $E$. coli. In one case there was an insertion and in the other case a deletion of DNA sequences.

The insertion present in two independently isolated plasmids was found to be IS2. In both cases IS2 was inserted near the $5^{\prime}$ end of the $\arg B$ gene, but in two different sites. This result is analogous to those obtained for other fungal genes cloned in E. coli (Walz et al., 1978; Brennan \& Struhl, 1980). In these examples, as well as in our case, IS2 was in orientation II relative to the activated gene. IS 2 located upstream of a gene and in orientation II is known to be responsible for switching on or increasing the level of transcription of many bacterial genes (Kleckner, 1981; Glansdorff et al., 1981). IS 2 has been postulated to be a donor of the -35 element of the prokaryotic promoter (Jaurin \& Nomark, 1983). Although we did not establish rigorously that IS2 is the source of the efficient promoter of the $A$. nidulans $\arg B$ gene in $E$. coli, this seems to be a plausible interpretation. In plasmids containing IS2 the $\arg B$ gene is situated such that it cannot be transcribed from any of the promoters identified in the vector (Stüber \& Bujard, 1981; Queen \& Rosenberg, 1981).

The second DNA rearrangement studied was identified as a deletion of DNA sequences adjacent to the $5^{\prime}$ end of the $\arg B$ gene. Transcription of the $\arg B$ gene was dependent on the $\mathrm{Tc}^{\mathrm{R}}$ gene promoter of the vector. In vitro experiments suggested, however, that this promoter can be used for transcription of the $\arg B$ gene not only in the plasmid with the deletion but also in the parental plasmid which is unable to suppress the OTCase deficiency in $E$. coli. Therefore the deletion seems to affect post-transcriptional steps of $\arg B$ gene expression. One possibility is that it results in an increase of the stability of the mRNA for OTCase. A second possibility is that the deletion affects the efficiency of translation of the $\arg B$ gene. Sequence analysis indicated that the deletion created a prokaryotic ribosome-binding site at the junction between the $A$. nidulans and the vector DNA. This site is located 81 nucleotides upstream of the codon postulated to initiate translation of the $\arg B$ gene in $A$. nidulans. If this putative ribosomebinding site is used in $E$. coli, the OTCase present in bacteria would be about $3 \mathrm{kDa}$ larger than that synthesized in $A$. nidulans. The difference in size between the OTCase isolated from $E$. coli cells and $A$. nidulans mycelium could be even greater because the $A$. nidulans enzyme is known to be located in mitochondria (unpublished results) and is probably truncated during transport through the mitochondrial membrane, as are many other mitochondrial proteins (Schatz \& Butow, 1983), including a human OTCase (Horwich et al., 1984). The results presented in this paper show that the OTCase synthesized in $E$. coli minicells is about $4 \mathrm{kDa}$ larger than that isolated from $A$. nidulans mycelium. This difference is consistent with the expected one and suggests that the putative ribosome-binding site created by the deletion is used for the initiation of translation of the $\arg B$ gene in $E$. coli.

The results presented in this paper show that there are several barriers preventing the efficient expression of the $\arg B$ gene in $E$. coli. One obstacle is the lack of proper signals for transcription of the $\arg B$ gene. This could be overcome by using prokaryotic promoters on the vector or introduced by IS2. Other barriers are not so strong, because in plasmids with an IS 2 insertion the increased efficiency of the transcription is enough to make the $\arg B$ gene functional. Nevertheless, transcription from the $T c^{R}$ gene promoter is not sufficient for the effective expression of this gene and an increase in translation efficiency is also necessary.

We thank Dr E. Bartnik for reading the manuscript. This work was supported by a grant from the Polish Academy of Sciences (09.7.2).

\section{REFERENCES}

BEGGS, J. D. B. (1978). Transformation of yeast by a replicating hybrid plasmid. Nature, London 275, 104-109.

Berse, B., Dmochowska, A., SkrzypeK, M.,
Węgleński, P., Bates, M. A. \& Weiss, R. L. (1983). Cloning and characterization of the ornithine carbamoyltransferasse gene from Aspergillus nidulans. Gene 25, 109-117. 
BonNER, W. M. \& LASKey, R. A. (1974), A film detection method for tritium labelled proteins and nucleic acids in polyacrylamide gels. European Journal of Biochemistry 46, 83-88.

Brennan, M. B. \& STRuHL, K. (1980). Mechanism of increasing expression of a yeast gene in Escherichia coli. Journal of Molecular Biology 136, 333-338.

Calos, M. P. \& Miller, J. H. (1980). Transposable elements. Cell 20, 579-595.

Clewell, D. B. \& HelinsKi, D. R. (1969). Supercoiled circular DNA-protein complex in Escherichia coli: purification and induced conversion to an open circular DNA form. Proceedings of the National Academy of Sciences of the United States of America 62, 1159-1166.

CosLoY, S. D. \& OishI, M. (1973). The nature of the transformation process in Escherichia coli $\mathrm{K}-12$. Molecular and General Genetics 124, 1-10.

Crabeel, M., Charlier, D., Cunin, R. \& GlansDorFF, N. (1979). Cloning and endonuclease restriction analysis of $\arg F$ and of the control region of the $\operatorname{argECBH}$ bipolar operon in Escherichia coli. Gene 5, 207-231.

Cybis, J., Piotrowska, M. \& Wg̨gleński, P. (1972). Genetic control of the arginine pathways in Aspergillus nidulans. Common regulation of anabolism and catabolism. Molecular and General Genetics 118, 273277.

DAgert, H. \& Ehrlich, S. (1979). Prolonged incubation in calcium chloride improves the competence of Escherichia coli cells. Gene 6, 23-28.

Ghosal, D., Sommer, H. \& Seadler, H. (1979). Nucleotide sequence of the transposable DNA element IS2. Nucleic Acids Research 6, 1111-1122.

GlansdorfF, N., Charlier, D. \& Zafarullah, M. (1981). Activation of gene expression by IS2 and IS3. Cold Spring Harbor Symposia on Quantitative Biology 45, 153-156.

Hinnen, A., Hicks, J. B. \& Fink, G. R. (1978). Transformation of yeast. Proceedings of the National Academy of Sciences of the United States of America 75, 1929-1933.

Horwich, A. L., Fenton, W. A., Williams, K. R., Kalousek, F., Kraus, J. P., Doolittle, R. F., Konisberg, W. \& Rosenberg, L. E. (1984). Structure and expression of a complementary DNA for the nuclear coded precursor of human mitochondrial ornithine transcarbamylase. Science 224, 1068-1974.

JAURIN, B. \& NOMARK, S. (1983). Insertion of IS2 creates a novel $a m p C$ promoter in Escherichia coli. Cell 32, 809-816.

KLECKNER, N. (1981). Transposable elements in procaryotes. Annual Review of Genetics 15, 341-404.

KOZAK, M. (1983). Comparison of initiation of protein synthesis in procaryotes, eucaryotes and organelles. Microbiological Reviews 47, 1-45.

LAEMMLI, U. K. (1970). Cleavage of structural proteins during the assembly of the head of bacteriophage $T 4$. Nature, London 227, 680-685.

LEE, F. \& YANOFSKY, C. (1977). Transcription termination at the $t r p$ operon attenuators of Escherichia coli and Salmonella typhimurium: RNA secondary structure and regulation of termination. Proceedings of the National Academy of Sciences of the United States of America 74, 4365-4369.
LeHRaCH, H., Diamond, D., WOZNEY, J. M. \& BOEDTKER, H. (1977). RNA molecular weight determination by gel electrophoresis under denaturing conditions, a critical reexamination. Biochemistry 16, 4743-4751.

Mackey, J. V., Brackman, K. H., Green, M. R. \& GREEN M. (1977). Preparation and characterization of highly radioactive in vitro labelled adenovirus DNA and DNA restriction fragments. Biochemistry 16, 4478-4483.

MaXam, A. M. \& Gilbert, W. (1977). A new method for sequencing DNA. Proceedings of the National Academy of Sciences of the United States of America 74, 560-564.

MaXam, A. M. \& GilberT, W. (1980). Sequencing endlabelled DNA with base-specific chemical cleavages. Methods in Enzymology 65, 499-560.

MilleR, J. H. (1972). Experiments in Molecular Genetics. Cold Spring Harbor, New York: Cold Spring Harbor Laboratory.

QUEEN, C. \& RosenberG, M. (1981). A promoter of pBR322 activated by a cAMP receptor protein. Nucleic Acids Research 14, 3365-3377.

REEVE, J. (1979). Use of minicells for bacteriophagedirected polypeptide synthesis. Methods in Enzymo$\log y$ 68, 493-503.

Roozen, K. J., Fenwick, R. G. \& Curtiss, R., III (1971). Synthesis of ribonucleic acid and protein in plasmid-containing minicells of Escherichia coli $\mathrm{K} 12$. Journal of Bacteriology 107, 21-23.

Schatz, G. \& Butow, R. A. (1983). How are proteins imported into mitochondria? Cell 32, 316-318.

Soberon, X., Covarrubias, L. \& Bolivar, F. (1980). Construction and characterization of new cloning vehicles. IV. Deletion derivatives of pBR322 and pBR325. Gene 9, 287-305.

SOUTHERN, E. M. (1975). Detection of specific sequences among DNA fragments separated by gel electrophoresis. Journal of Molecular Biology 98, 503-517.

STÜBER, D. \& BUJARD, H. (1981). Organization of transcriptional signals in plasmids pBR322 and pACYC184. Proceedings of the National Academy of Sciences of the United States of America 78, 168-171.

SUTCLIFFE, J. G. (1979). Complete nucleotide sequence of the Escherichia coli plasmid pBR322. Cold Spring Harbor Symposia on Quantitative Biology 43, 77-79.

Thomas, P. S. (1980). Hybridization of denatured RNA and small DNA fragments transferred to nitrocellulose. Proceedings of the National Academy of Sciences: of the United States of America 77, 52015205.

Walz, A., Ratzkin, B. \& Carbon, J. (1978). Control of expression of a cloned yeast (Saccharomyces cerevisice) gene (trp5) by a bacterial insertion element (IS2). Proceedings of the National Academy of Sciences of the United States of America 75, 61726176.

ZabeaU, M. \& Stanley, K. K. (1982). Enhanced expression of cro- $\beta$-galactosidase fusion protein under the control of the $\mathrm{P}_{\mathrm{R}}$ promoter of bacteriophage $\lambda$. EMBO Journal 1, 1217-1224. 